\title{
Three-Dimensional Mesoporous Covalent Organic Frameworks through Steric Hindrance Engineering
}

\author{
Yujie Wang, ${ }^{\dagger} \S$ Yaozu Liu ${ }^{\dagger, \S}$ Hui Li, ${ }^{\dagger}$ Xinyu Guan, ${ }^{\dagger}$ Ming Xue ${ }^{\dagger}$ Yushan Yan,${ }^{\dagger}$ Valentin Valtchev, ${ }^{\dagger}, \|$ \\ Shilun Qiu, ${ }^{\dagger}$ and Qianrong Fang, ${ }^{* \dagger}$ \\ ${ }^{\dagger}$ State Key Laboratory of Inorganic Synthesis and Preparative Chemistry, Jilin University, Changchun 130012, P. R. China \\ Department of Chemical and Biomolecular Engineering, Center for Catalytic Science and Technology, University of \\ Delaware, Newark, DE 19716, USA
}

" Normandie Université, ENSICAEN, UNICAEN, CNRS, Laboratoire Catalyse et Spectrochimie, 14000 Caen, France

Supporting Information Placeholder

\begin{abstract}
The development of three-dimensional covalent organic frameworks (COFs) with large pores and high surface areas is of great importance for various applications. However, it remains a major challenge due to the unavoidable structural interpenetration and pore collapse after the removal of guest species sitiated in the pores. Herein, we report for the first time a series of 3D mesoporous COFs through a general strategy of enhanced steric hindrance. By using methoxy-modified monomer and increasing methyl groups of linkers, these 3D COFs can be obtained successfully as exclusively non-interpenetrated diamondoid structures, permanent mesopores (up to $26.5 \AA$ ), and high surface areas (>3000 $\mathrm{m}^{2} \mathrm{~g}^{-1}$ ), which are far superior to those of reported conventional COFs with the same topology. This work thus opens a way to create 3D large-porous COFs for potential applications in adsorption and separation of large inorganic, organic, and biological species.
\end{abstract}

Covalent organic frameworks (COFs) are fascinating crystalline porous polymers composed of light elements (typically $\mathrm{H}, \mathrm{B}, \mathrm{C}$, $\mathrm{N}$, and $\mathrm{O}$ ) and linked by reversible covalent bonds. ${ }^{1-4}$ They feature well-defined pores and high specific surface areas and thus hold great promises in a variety of potential uses, such as adsorption and separation, ${ }^{5-7}$ organic electronics, ${ }^{8-12}$ heterogeneous catalysis, ${ }^{13-17}$ and some others. ${ }^{18-23}$ Over the past decade, most of COF materials, however, were exploited based on two-dimensional (2D) structures with characteristic eclipsed AA stacking. Three-dimensional (3D) COFs have recently attracted widespread attentions owing to their unique pores and excellent properties. ${ }^{24-32}$ Notably, the urgent issues emerge for the construction of 3D large-porous COFs, including inevitable structural interpenetration for restricting the pore size and pore shrinkage or collapse after the guest removal. For example, Wang and Zhang et al. have proven the reversible crystal-transformation and dynamic response in an interpenetrating 3D COF, LZU-301, which has pore size stretchable from $5.8 \times 10.4 \AA$ to $9.6 \times 10.4 \AA$ by the induction of guests. ${ }^{33}$ Recently, by extending the linear linkers, we obtained the most interpenetrated framework for 3D COFs reported, 3D-IL-COF-3 with 11-fold interpenetrated net, exhibiting a small pore size of $12.4 \AA$ and relatively low specific surface area of $870 \mathrm{~m}^{2} / \mathrm{g} .{ }^{34}$ Thus, the expansion of 3D COFs with
Scheme 1. Strategy for preparing 3D mesoporous $\mathrm{COFs}^{\mathrm{a}}$

a)

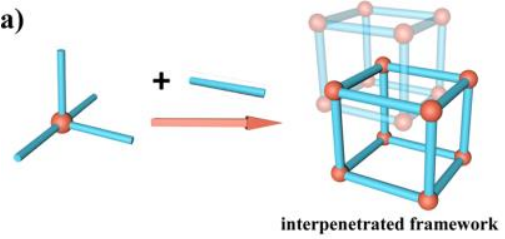

b)

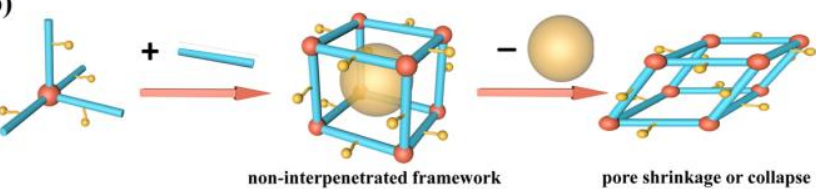

c)

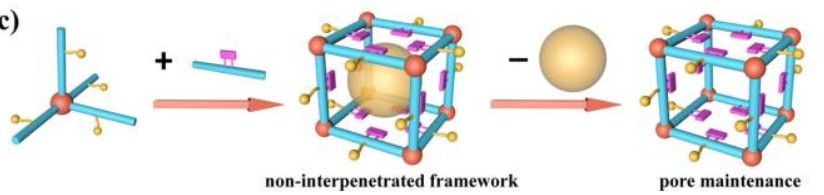

a) The interpenetrated microporous framework constructed from undecorated edges. (b) The non-interpenetrated mesoporous framework built up from partially decorated edges, in which the pore shrinkage or collapse will happen after the guest removal. (c) The non-interpenetrated mesoporous framework created by highly decorated edges, in which the pores can be maintained entirely after the guest removal.

large pores and high surface areas is of primary importance but remains a tremendouschallenge.

Herein, we report a series of 3D mesoporous COFs (3D-Meso-COFs) with unusual non-interpenetrated diamondoid (dia) topology based on the inovative enhanced steric hindrance strategy. By employing methoxy-modified monomer and changing substituents (methyl groups) of linkers, these 3D-Meso-COFs can be turned successfully from pore shrinkage to partial and full pore maintenance. Remarkably, one of the obtained COF material shows permanent mesopore with a size of $26.5 \AA$ and impressive specific surface area of $3023 \mathrm{~m}^{2} \mathrm{~g}^{-1}$, which are much higher than that of the convention interpenetrating $\mathrm{COF}$ with the same network. To the best of our knowledge, this study 
represents the first case of 3D mesoporous COF with non-interpenetrated dia topology.

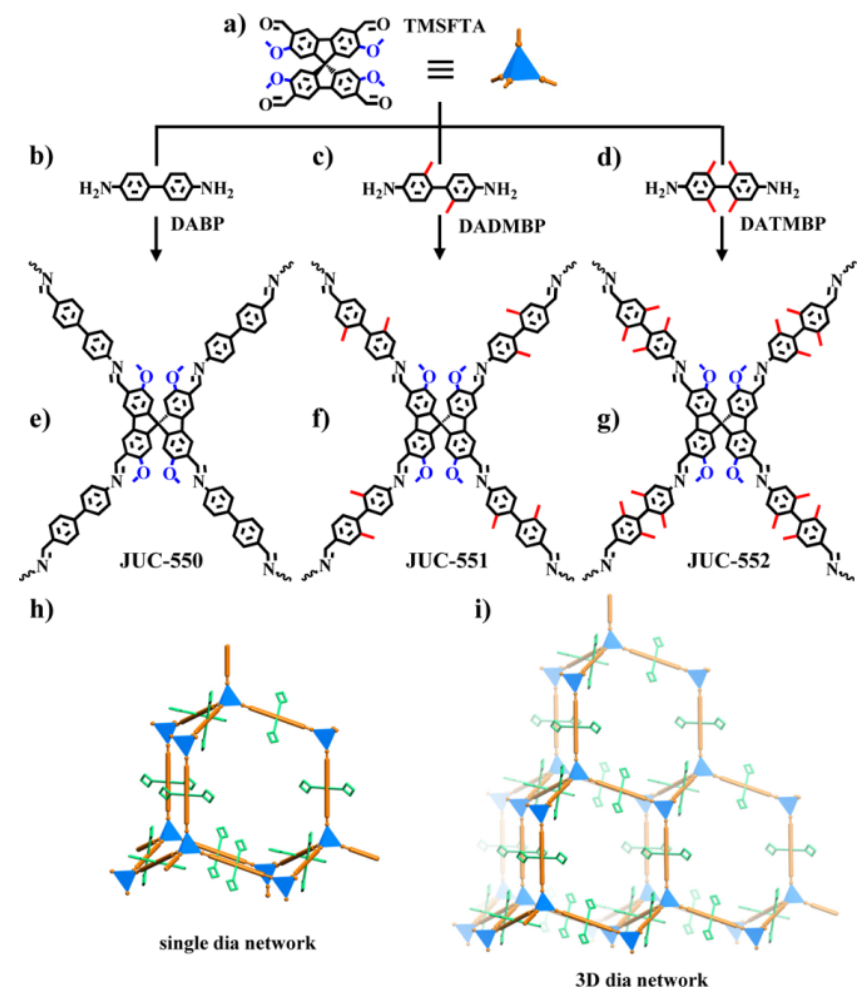

Figure 1. (a-d) Molecular structures of TMSFTA (a) as a tetrahedral building unit and DABP (b), DADMBP (c) and DATMBP (d) as a linear building unit, respectively. (e-g) 3D mesoporous COFs, denoted as JUC-550 to -552 , constructed by the condensation reaction of TMSFTA and DABP, DADMBP or DATMBP. (h and i) Single dia network $(\mathrm{h})$ and $3 \mathrm{D}$ non-interpenetrated dia structure (i) with decorated edges in these obtained COFs.

Our strategy for the preparation of 3D-Meso-COFs is based on the steric hindrance by employing various linkers. As shown in Scheme 1three possible situations can occur: (a) the interpenetrated microporous framework constructed from undecorated edges; (b) the non-interpenetrated mesoporous framework built up from partially decorated edges, in which the pore shrinkage or collapse will happen after the guest removal; and (c) the non-interpenetrated mesoporous framework created by highly decorated edges, in which the pores can be maintained entirely after the removal of guests. To implement this strategy, we firstly designed a novel methoxy-modified monomer, 2,2',7,7'-tetramethoxy-9, $9^{\prime}$-spirobi[fluorene]-3,3',6,6'-tetracarbald ehyde (TMSFTA), as a tetrahedral building unit (Figure 1a). Further, we chose three linear linkers, 4,4'-diaminobiphenyl (DABP, Figure 1b) without any substituents, 4,4'-diamino-2,2'-dimethylbiphenyl (DADMBP, Figure 1c) with two methyl groups, and 4,4'-diaminp-2,2',6,6'-tetramethylbiphenyl (DATMBP, Figure 1d) with four methyl groups. The condensation of TMSFTA and DABP, DADMBP or DATMBP produced a series of 3D COFs, termed JUC-550, JUC-551 and JUC-552 respectively $(\mathrm{JUC}=$ Jilin University China, Figure 1e-g). In light of the linking of tetrahedral and linear building units and their steric hindrance, these structures are expected to be the non-interpenetrated dia nets (Figure $1 \mathrm{~h}$ and 1i). ${ }^{35}$
Typically, the syntheses were carried out by suspending TMSFTA with DABP, DADMBP or DATMBP into the solvent of dioxane or mesitylene in the presence of acetic acid, followed by heating at $120{ }^{\circ} \mathrm{C}$ for 3 days to give crystalline solids at high yields of $85 \%$ for JUC-550, $83 \%$ for JUC-551, and $87 \%$ for JUC-552, respectively. These products were stable in various organic solvents, such as acetone, hexanes, tetrahydrofuran (THF), $N, N$-dimethylformamide (DMF), $N$-methyl-2-pyrrolidone (NMP), and dimethyl sulfoxide (DMSO).
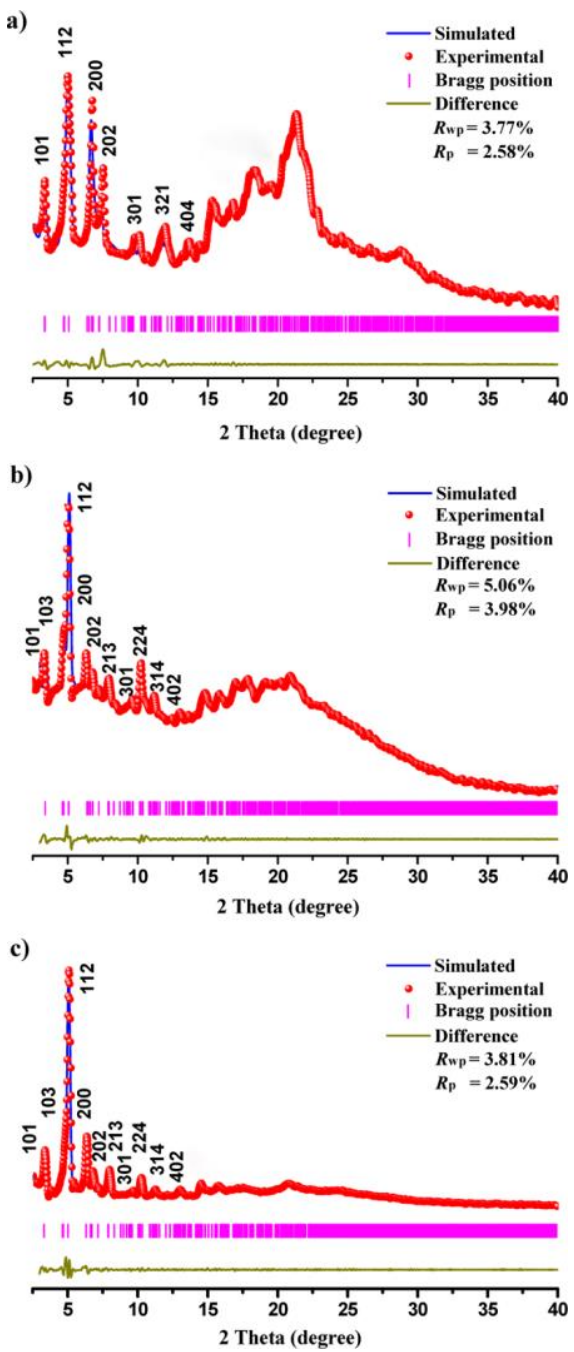

Figure 2. PXRD patterns of JUC-550 (a), JUC-551 (b) and JUC-552 (c).

The structural characteristics of new COFs were determined by the complementary method. The crystal morphology was observed by scanning electron microscopy (SEM), which shows isometric crystals (Figures S1-3). Fourier transform infrared (FT-IR) spectra exhibited new peaks around $1617 \mathrm{~cm}^{-1}$ for JUC-550, $1612 \mathrm{~cm}^{-1}$ for JUC-551, and $1617 \mathrm{~cm}^{-1}$ for JUC-552, corresponding to typical $\mathrm{C}=\mathrm{N}$ stretching vibrations (Figures $\mathrm{S} 4-6$ ). Furthermore, the solid state ${ }^{13} \mathrm{C}$ cross-polarization magic-angle-spinning (CP/MAS) NMR spectra confirmed the presence of carbon from the $\mathrm{C}=\mathrm{N}$ bond at $158 \mathrm{ppm}$ for JUC-550, $161 \mathrm{ppm}$ for JUC-551, and $159 \mathrm{ppm}$ for JUC-552, respectively (Figures S7-9). According to the thermogravimetric analysis (TGA), these COFs were thermally stable up to $350{ }^{\circ} \mathrm{C}$ under nitrogen (Figures S10-12). 

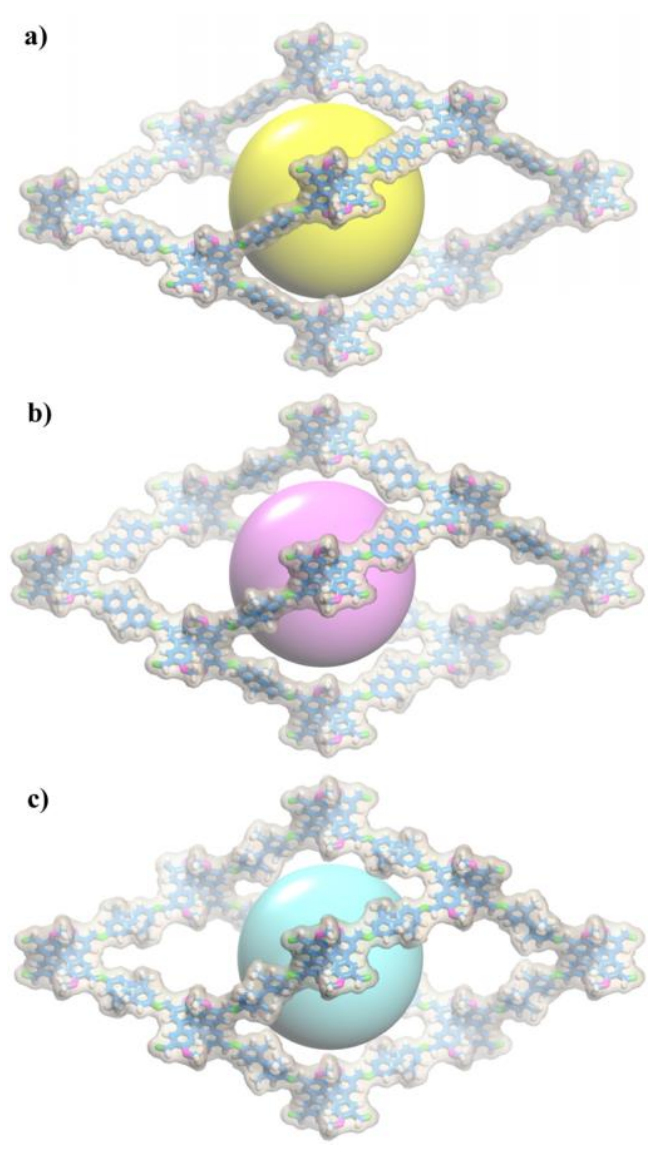

Figure 3. Structural representations of JUC-550 (a), JUC-551 (b), and JUC-552 (c). C, blue; H, gray; N, green; O, pink. The spheres with different colors represent the pores in 3D-Meso-COFs.

The structures of 3D-Meso-COFs were resolved by powder $\mathrm{X}$-ray diffraction (PXRD) combined with structural simulations (Figure 2). After a geometrical energy minimization by using the Materials Studio software package ${ }^{36}$ based on the non-interpenetrated dia net, the unit cell parameters were obtained $\left(a=b=27.5727 \AA, c=74.5020 \AA\right.$ and $\alpha=\beta=\gamma=90^{\circ}$ for JUC-550; $a=b=27.8446 \AA, c=74.6490 \AA$ and $\alpha=\beta=\gamma=90^{\circ}$ for JUC-551; and $a=b=27.8435 \AA, c=74.6423 \AA$ and $\alpha=\beta=\gamma$ $=90^{\circ}$ for JUC-552). The simulated PXRD patterns were in good agreement with the experimental results. Full profile pattern matching (Pawley) refinements were carried out on the experimental data from solvated samples. Peaks at 3.35, 4.98, $6.74,7.53,10.07,11.96$ and $13.65^{\circ}$ for JUC-550 correspond to the (101), (112), (200), (202), (301), (321) and (404) Bragg peaks of space group $I 44_{1} / \mathrm{a}$ (No. 88); peaks at 3.38, 4.79, 5.08, 6.37, 6.82, $8.01,9.71,10.29,11.23$ and $13.07^{\circ}$ for JUC-551 correspond to the (101), (103), (112), (200), (202), (213), (301), (224), (314) and (402) Bragg peaks of the same space group; and peaks at 3.34, $4.78,5.07,6.35,6.79,7.99,9.68,10.23,11.29$ and $13.01^{\circ}$ for JUC-552 correspond to the (101), (103), (112), (200), (202), (213), (301), (224), (314) and (402) Bragg peaks of the same space group, respectively. The refinement results yielded unit cell parameters nearly equivalent to the predictions with good agreement factors $(R \mathrm{p}=2.58 \%$ and $\omega R \mathrm{p}=3.77 \%$ for JUC-550; $R \mathrm{p}=3.98 \%$ and $\omega R \mathrm{p}=5.06 \%$ for JUC-551; and $R \mathrm{p}=2.59 \%$ and $\omega R \mathrm{p}=3.81 \%$ for JUC-552). Some alternative structures, such as 2- and 3-fold interpenetrated dia nets, were also set up; however, their simulated PXRDs did not match to the experimental ones (Figures S13-24). Thus, the obtained COFs were proposed to have the expected mesoporous frameworks with non-interpenetrated dia topology (Figure 3).

It should be noted that some peaks after $2 \theta>15^{\circ}$ in JUC-550 are intense, while those of JUC-551 showed low intensity. Most important, the corresponding peaks of JUC-552 were very week revealing the impact of the linker on the crystalline framework. Furthermore, after activation under vacuum at $100{ }^{\circ} \mathrm{C}$ for $12 \mathrm{~h}$, the first peak $\left(2 \theta=3.35^{\circ}\right)$ was disappeared in JUC-550, while showed a slight variation in JUC-551. However, JUC-552 exhibited enhanced intensity for its first peak $\left(2 \theta=3.34^{\circ}\right)$, which means its mesopores have been well retained. Notably, this process can be repeated after immersing these samples into organic solvents, such as acetone (Figures S25-27). These results demonstrated clearly that a guest-induced reversible crystal-structure transformation took place in JUC-550; ${ }^{33}$ however, the linker with high substituents, DATMBP, gives rise to JUC-552 with increased structural rigidity due to the strong steric hindrance, and thus can effectively eliminate the dynamic behavior of frameworks. ${ }^{37}$
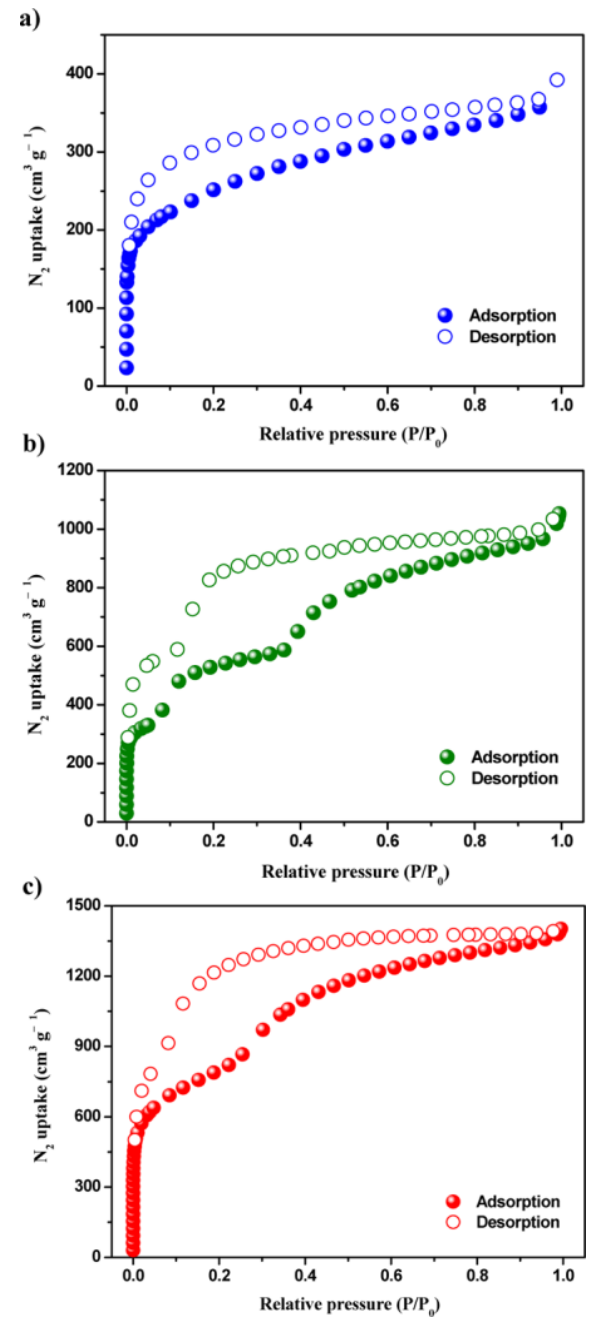

Figure 4. $\mathrm{N}_{2}$ adsorption-desorption isotherms for JUC-550 (a), JUC-551 (b) and JUC-552 (c).

The influence of steric hindrance in activated 3D-Meso-COFs was further proved by nitrogen $\left(\mathrm{N}_{2}\right)$ adsorption-desorption measurment at $77 \mathrm{~K}$. As can be seen in Figure 4a, a rapid uptake at low relative pressure characteristic of micropore material was observed for JUC-550, which reveals a pore shrinkage. JUC-551 showed a rapid uptake at a low pressure of $\mathrm{P} / \mathrm{P}_{0}<0.05$, followed 
by two other steps at $\mathrm{P} / \mathrm{P}_{0}=0.1$ and 0.4 , which means its partial pore shrinkage and the presence of different pores. On the contrary, JUC-552 had a high uptake at a low pressure of $\mathrm{P} / \mathrm{P}_{0}<$ 0.05 , followed by well define second step at $\mathrm{P} / \mathrm{P}_{0}=0.3$, which is a typical characteristic of mesoporous materials. All curves of 3D-Meso-COFs showed a desorption hysteresis, which can be attributed to the effect of substituents and textural mesopores from the agglomeration of COF microcrystals. ${ }^{38}$ The Brunauer-Emmett-Teller (BET) equation was employed to evaluate the specific surface area of the series of COF materials. A strong correlation between the linker employed and the $S_{\text {BET }}$ was found, thus JUC-550, JUC-551 and JUC-552 showed $846 \mathrm{~m}^{2}$ $\mathrm{g}^{-1} 1728 \mathrm{~m}^{2} \mathrm{~g}^{-1}$, and $3023 \mathrm{~m}^{2} \mathrm{~g}^{-1}$, respectively. Furthermore, their pore-size distributions calculated by nonlocal density functional theory (NLDFT) demonstrated the micropores with the size of $10.6 \AA$ for JUC-550 and two kinds of pores with the sizes of 14.0 $\AA$ and $22.0 \AA$ for JUC-551. Notably, JUC-552 showed the mesopores with the dominant size of $26.5 \AA$, which is in good agreement with that of the proposed model $(26.0 \AA)$, and thus indicated that the pores can be maintained after the guest removal.

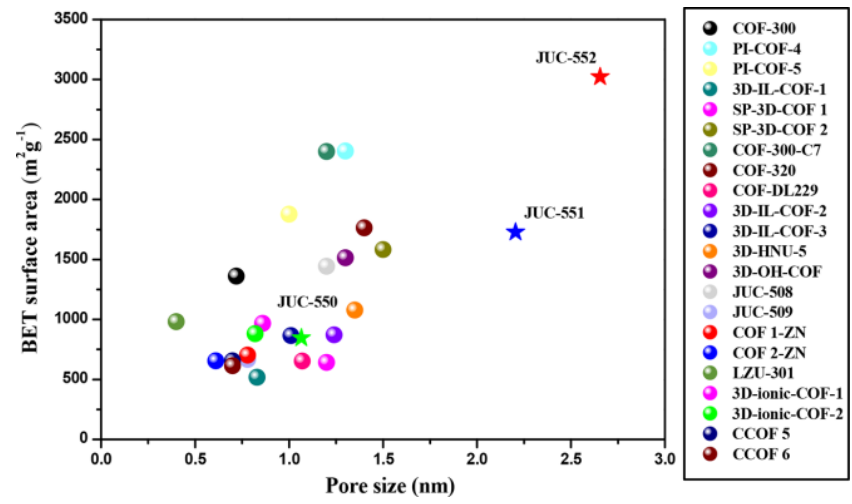

Figure 5. A plot of BET surface area against pore size in reported COFs with dia topology.

As a comparison, we summarized the BET surface areas and pore sizes of current reported COFs with dia topology in Figure 5 and Table S1. Remarkably, the pore size of JUC-552 (26.5 $\AA$ ) is far superior to those of reported materials, such as $4.0 \AA$ for COF-300-C7, ${ }^{39} 12.0 \AA$ for COF-320, ${ }^{40} 13.0 \AA$ for JUC-508, ${ }^{32}$ and $13.5 \AA$ for $3 \mathrm{D}-\mathrm{OH}-\mathrm{COF} .{ }^{31}$ The BET surface area of JUC-552 $\left(3023 \mathrm{~m}^{2} \mathrm{~g}^{-1}\right)$ is also much higher than those of reported conventional COFs, such as $517 \mathrm{~m}^{2} \mathrm{~g}^{-1}$ for 3D-IL-COF-1, ${ }^{34} 864$ $\mathrm{m}^{2} \mathrm{~g}^{-1}$ for 3D-HNU-5, ${ }^{41} 1762 \mathrm{~m}^{2} \mathrm{~g}^{-1}$ for COF-DL229, ${ }^{42}$ and 2403 $\mathrm{m}^{2} \mathrm{~g}^{-1}$ for PI-COF-4. ${ }^{38}$ Undoubtly, these data revealed that the enhanced steric hindrance is a promising strategy for the construction of COF materials with large pores and high specific surface areas.

To further demonstrate the mesopores in 3D-Meso-COFs, we explored the inclusion of a large dye molecule, Rh6G, with a size of $16.0 \AA$. Typically, the samples of 3D-Meso-COFs were immersed in a solution of Rh6G $(0.1 \mathrm{mM})$. The amount of Rh6G in the supernatant was monitored through UV-Vis spectrophotometry, and the characteristic absorbance at $526 \mathrm{~nm}$ was measured over a period of 60 mins. As showed in Figures S36 and S37, the results displayed a continuous decrease in the amount of Rh6G in solvated 3D-Meso-COFs due to their maintained mesopores. As a control, a similar experiment was carried out with COF-320, whose pore size (12.0 $\AA$ ) is smaller than Rh6G. the experiment confirmed the absence of adsorption Rh6G on COF-320, no obvious reduction in absorbance was found (Figures S36 and S37).
In conclusion, we design and synthesize a series of $3 \mathrm{D}$ mesoporous COFs by a simple and effective strategy of enhanced steric hindrance. By utilizing methoxy-modified linker, 3D-Meso-COFs can be prepared as non-interpenetrated dia structures. The new strategy allows fine tuning the properties of COF mesoporosity by controlling the amount of substutient in the framework. Thus a new material, JUC-552 with stable mesopores ( $26.5 \AA$ ) and outstanding specific surface areas (up to $3023 \mathrm{~m}^{2}$ $\mathrm{g}^{-1}$ ), which are much higher than those of reported COFs with the same topology, was obtained. This research starts a new avenue to construct 3D large-porous COFs for various applications, such as adsorption and separation of biological, inorganic, and organic molecules with large sizes.

\section{ASSOCIATED CONTENT}

\section{Supporting Information}

Synthetic procedures, SEM, FTIR, solid state ${ }^{13} \mathrm{C}$ NMR, TGA, BET plots, and pore size distribution. This material is available free of charge via the internet at http://pubs.acs.org.

\section{AUTHOR INFORMATION}

\section{Corresponding Author}

*qrfang@jlu.edu.cn

\section{Author Contributions}

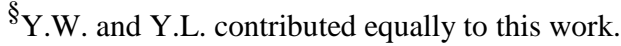

Notes

The authors declare no competing financial interests.

\section{ACKNOWLEDGMENT}

This work was supported by National Natural Science Foundation of China $(21571079,21621001,21390394,21571076$, and 21571078), "111" project (B07016 and B17020), and the program for JLU Science and Technology Innovative Research Team. V.V. and Q.F. acknowledge the Thousand Talents program (China).

\section{REFERENCES}

(1) Côté, A. P.; Benin, A. I.; Ockwig, N. W.; O'Keeffe, M.; Matzger, A. J.; Yaghi, O. M. Porous, Crystalline, Covalent Organic Frameworks. Science 2005, 310, 1166.

(2) Feng, X.; Ding, X. S.; Jiang, D. L. Covalent Organic Frameworks. Chem. Soc. Rev. 2012, 41, 6010.

(3) Ding, S. Y.; Wang, W. Covalent Organic Frameworks (COFs): from Design to Applications. Chem. Soc. Rev. 2013, 42, 548.

(4) Diercks, C. S.; Yaghi, O. M. The Atom, the Molecule, and the Covalent Organic Framework. Science 2017, 355, eaal1585.

(5) Han, S. S.; Furukawa, H.; Yaghi, O. M.; Goddard, W. A., III Covalent Organic Frameworks as Exceptional Hydrogen Storage Materials. J. Am. Chem. Soc. 2008, 130, 11580.

(6) Kuhn, P.; Antonietti, M.; Thomas, A. Porous, Covalent TriazineBased Frameworks Prepared by Ionothermal Synthesis. Angew. Chem., Int. Ed. 2008, 47, 3450.

(7) Wang, S.; Wang, Q.; Shao, P.; Han, Y.; Gao, X.; Ma, L.; Yuan, S.; Ma, X.; Zhou, J.; Feng, X.; Wang, B. Exfoliation of Covalent Organic Frameworks into Few-layer Redox-Active Nanosheets as Cathode Materials for Lithium-Ion Batteries. $J$. Am. Chem. Soc. 2017, 139, 4258.

(8) Wan, S.; Guo, J.; Kim, J.; Ihee, H.; Jiang, D. A Belt-Shaped, Blue Luminescent, and Semiconducting Covalent Organic Framework. Angew. Chem., Int. Ed. 2008, 47, 8826. 
(9) Dogru, M.; Handloser, M.; Auras, F.; Kunz, T.; Medina, D.; Hartschuh, A.; Knochel, P.; Bein, T. A Photoconductive Thienothiophene-Based Covalent Organic Framework Showing Charge Transfer Towards Included Fullerene. Angew. Chem., Int. Ed. 2013, 52, 2920.

(10) Bertrand, G. H. V.; Michaelis, V. K.; Ong, T. C.; Griffin, R. G.; Dinca, M. Thiophene-Based Covalent Organic Frameworks. Proc. Natl. Acad. Sci. U. S. A. 2013, 110, 4923.

(11) Du, Y.; Yang, H.; Whiteley, J. M.; Wan, S.; Jin, Y.; Lee, S. H.; Zhang, W. Ionic Covalent Organic Frameworks with Spiroborate Linkage. Angew. Chem., Int. Ed. 2016, 55, 1737.

(12) Li, H.; Chang, J. H.; Li, S. S.; Guan, X. Y.; Li, D. H.; Li, C. Y.; Tang, L. X.; Xue, M.; Yan, Y. S.; Valtchev, V.; Qiu, S. L.; Fang, Q. R. Three-Dimensional Tetrathiafulvalene-Based Covalent Organic Frameworks for Tunable Electrical Conductivity. J. Am. Chem. Soc. 2019, 141, 13324.

(13) Ding, S. Y.; Gao, J.; Wang, Q.; Zhang, Y.; Song, W. G.; $\mathrm{Su}$, C. Y.; Wang, W. Construction of Covalent Organic Framework for Catalysis: Pd/COF-LZU1 in Suzuki-Miyaura Coupling Reaction. J. Am. Chem. Soc. 2011, 133, 19816.

(14) Peng, Y.; Hu, Z.; Gao, Y.; Yuan, D.; Kang, Z.; Qian, Y.; Yan, N.; Zhao, D. Synthesis of a Sulfonated Two-Dimensional Covalent Organic Framework as an Efficient Solid Acid Catalyst for Biobased Chemical Conversion. ChemSusChem 2015, 8, 3208.

(15) Vyas, V. S.; Haase, F.; Stegbauer, L.; Savasci, G.; Podjaski, F.; Ochsenfeld, C.; Lotsch, B. V. A Tunable Azine Covalent Organic Framework Platform for Visible Light-Induced Hydrogen Generation. Nat. Commun. 2015, 6, 8508.

(16) Sun, Q.; Aguila, B.; Perman, J.; Nguyen, N.; Ma, S. Q. Flexibility Matters: Cooperative Active Sites in Covalent Organic Framework and Threaded Ionic Polymer. J. Am. Chem. Soc. 2016, $138,15790$.

(17) Wang, X.; Han, X.; Zhang, J.; Wu, X.; Liu, Y.; Cui, Y. Homochiral 2D Porous Covalent Organic Frameworks for Heterogeneous Asymmetric Catalysis. J. Am. Chem. Soc. 2016, $138,12332$.

(18) Oh, H.; Kalidindi, S. B.; Um, Y.; Bureekaew, S.; Schmid, R.; Fischer, R. A.; Hirscher, M. A Cryogenically Flexible Covalent Organic Framework for Efficient Hydrogen Isotope Separation by Quantum Sieving. Angew. Chem., Int. Ed. 2013, 52, 13219.

(19) Zhou, T. Y.; Xu, S. Q.; Wen, Q.; Pang, Z. F.; Zhao, X. One-Step Construction of Two Different Kinds of Pores in a 2D Covalent Organic Framework. J. Am. Chem. Soc. 2014, 136, 15885.

(20) Chandra, S.; Kundu, T.; Kandambeth, S.; BabaRao, R.; Marathe, M. Y.; Kunjir, S. M.; Banerjee, R. Phosphoric Acid Loaded Azo $(-\mathrm{N}=\mathrm{N}-)$ Based Covalent Organic Framework for Proton Conduction. J. Am. Chem. Soc. 2014, 136, 6570.

(21) Jin, E. Q.; Asada, M.; Xu, Q.; Dalapati, S.; Addicoat, M. A.; Brady, M. A.; Xu, H.; Nakamura, T.; Heine, T.; Chen, Q. H.; Jiang, D. L. Two-Dimensional sp2 Carbon-Conjugated Covalent Organic Frameworks. Science 2017, 357, 673.

(22) Guan, X. Y.; Li, H.; Ma, Y. C.; Xue, M.; Fang, Q. R.; Yan, Y. S.; Valtchev, V.; Qiu, S. L. Chemically Stable Polyarylether-Based Covalent Organic Frameworks. Nat. Chem. 2019, 11, 587.

(23) Guo, Z. B.; Zhang, Y. Y.; Dong, Y.; Li, J.; Li, S. W.; Shao, P. P.; Feng, X.; Wang, B. Fast Ion Transport Pathway Provided by Polyethylene Glycol Confined in Covalent Organic Frameworks. J. Am. Chem. Soc. 2019, 141, 1923.

(24) El-Kaderi, H. M.; Hunt, J. R.; Mendoza-Cortes, J. L.; Côté, A. P.; Taylor, R. E.; O'Keeffe, M.; Yaghi, O. M. Designed Synthesis of 3D Covalent Organic Frameworks. Science 2007, $316,268$.

(25) Uribe-Romo, F. J.; Hunt, J. R.; Furukawa, H.; Klöck, C.; O'Keeffe, M.; Yaghi, O. M. A Crystalline Imine-Linked 3D
Porous Covalent Organic Framework. J. Am. Chem. Soc. 2009, 131,4570 .

(26) Beaudoin, D.; Maris, T.; Wuest, J. D. Constructing Monocrystalline Covalent Organic Networks by Polymerization. Nat. Chem. 2013, 5, 830.

(27) Liu, Y. Z.; Ma, Y. H.; Zhao, Y. B.; Sun, X. X.; Gándara, F.; Furukawa, H.; Liu, Z.; Zhu, H. Y.; Zhu, C. H.; Suenaga, K.; Oleynikov, P.; Alshammari, A. S.; Zhang, X.; Terasaki, O.; Yaghi, O. M. Weaving of Organic Threads into a Crystalline Covalent Organic Framework. Science 2016, 351, 365.

(28) Li, H.; Pan, Q. Y.; Ma, Y. C.; Guan, X. Y.; Xue, M.; Fang, Q. R.; Yan, Y. S.; Valtchev, V.; Qiu, S. L. Three-Dimensional Covalent Organic Frameworks with Dual Linkages for Bifunctional Cascade Catalysis. J. Am. Chem. Soc. 2016, 138, 14783.

(29) Li, Z. L.; Li, H.; Guan, X. Y.; Tang, J. J.; Yusran, Y.; Li, Z.; Xue, M.; Fang, Q. R.; Yan, Y. S.; Valtchev, V.; Qiu, S. L. ThreeDimensional Ionic Covalent Organic Frameworks for Rapid, Reversible, and Selective Ion Exchange. J. Am. Chem. Soc. 2017, 139, 17771.

(30) Ma, T. Q.; Kapustin, E. A.; Yin, S. X.; Liang, L.; Zhou, Z. Y.; Niu, J.; Li, L. H.; Wang, Y. Y.; Su, J.; Li, J.; Wang, X. G.; Wang, W. D.; Wang, W.; Sun, J. L.; Yaghi, O. M. Single-Crystal $\mathrm{X}$-Ray Diffraction Structures of Covalent Organic Frameworks. Science 2018, 361, 48.

(31) Lu, Q. Y.; Ma, Y. C.; Li, H.; Guan, X. Y.; Yusran, Y.; Xue, M.; Fang, Q. R.; Yan, Y. S.; Qiu, S. L.; Valtchev, V. Postsynthetic Functionalization of Three-Dimensional Covalent Organic Frameworks for Selective Extraction of Lanthanide Ions. Angew. Chem., Int. Ed. 2018, 57, 6042.

(32) Yan, S. C.; Guan, X. Y.; Li, H.; Li, D. H.; Xue, M.; Yan, Y. S.; Valtchev, V.; Qiu, S. L.; Fang, Q. R. Three-dimensional Salphen-based Covalent-Organic Frameworks as Catalytic Antioxidants. J. Am. Chem. Soc. 2019, 141, 2920.

(33) Ma, Y. X.; Li, Z. J.; Wei, L.; Ding, S. Y.; Zhang, Y. B.; Wang, W. A Dynamic Three-Dimensional Covalent Organic Framework. J. Am. Chem. Soc. 2017, 139, 4995.

(34) Guan, X. Y.; Ma, Y. C.; Li, H.; Yusran, Y.; Xue, M.; Fang, Q. R.; Yan, Y. S.; Valtchev, V.; Qiu, S. L. Fast, Ambient Temperature and Pressure Ionothermal Synthesis of Three-Dimensional Covalent Organic Frameworks. J. Am. Chem. Soc. 2018, 140, 4494.

(35) Bonneau, C.; Delgado-Friedrichs, O.; O'Keeffe, M.; Yaghi, O. M. Three-Periodic Nets and Tillings: Minimal Nets. Acta Crystallogr., Sect. A: Found. Crystallogr. 2004, A60, 517.

(36) Materials Studio ver. 7.0; Accelrys Inc.: San Diego, CA.

(37) Yuan, S.; Zou, L. F.; Li, H. X.; Chen, Y. P.; Qin, J. S.; Zhang, Q.; Lu, W. G.; Hall, M. B.; Zhou, H. C. Flexible Zirconium Metal-Organic Frameworks as Bioinspired Switchable Catalysts. Angew. Chem., Int. Ed. 2016, 55, 10776.

(38) Fang, Q.; Wang, J.; Gu, S.; Kaspar, R. B.; Zhuang, Z.; Zheng, J.; Guo, H.; Qiu, S.; Yan, Y. 3D Porous Crystalline Polyimide Covalent Organic Frameworks for Drug Delivery. $J$. Am. Chem. Soc. 2015, 137, 8352.

(39) Ma, T. Q.; Li, J.; Niu, J.; Zhang, L.; Etman, A. S.; Lin, C.; Shi, D. E.; Chen, P. H.; Li, L. H.; Du, X.; Sun, J. L.; Wang, W. Observation of Interpenetration Isomerism in Covalent Organic Frameworks. J. Am. Chem. Soc. 2018, 140, 6763.

(40) Zhang, Y. B.; Su, J.; Furukawa, H.; Yun, Y. F.; Gándara, F.; Duong, A.; Zou, X. D.; Yaghi, O. M. Single-Crystal Structure of a Covalent Organic Framework. J. Am. Chem. Soc. 2013, 135, 16336.

(41) Guan, P. X.; Qiu, J. K.; Zhao, Y. L.; Wang, H. Y.; Li, Z. Y.; Shi, Y. L.; Wang, J. J. A Novel Crystalline Azine-Linked Three-Dimensional Covalent Organic Framework for $\mathrm{CO}_{2}$ Capture and Conversion. Chem. Commun. 2019, 55, 12459. 
(42) Wang, C.; Wang, Y.; Ge, R.; Song, X. D.; Xing, X. Q.; Jiang, Q. K.; Lu, H.; Hao, C.; Guo, X. W.; Gao, Y. N.; Jiang, D.

L. A 3D Covalent Organic Framework with Exceptionally High Iodine Capture Capability. Chem. Eur. J. 2018, 24, 585. 
TOC Graphic:

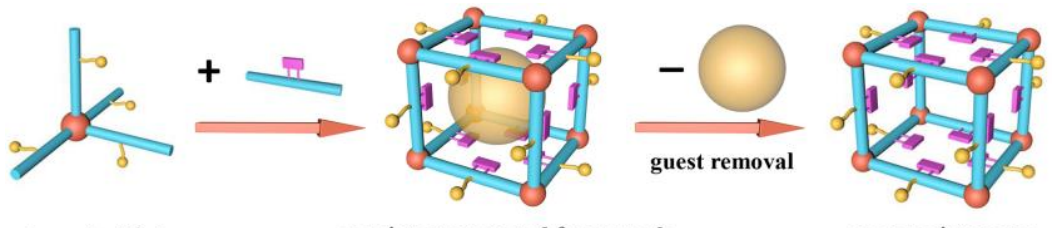

